Article

\title{
On Connection between Second-Degree Exterior and Symmetric Derivations of Kähler Modules
}

\author{
Hamiyet Merkepçi ${ }^{\mathbb{D}}$ \\ Department of Mathematics, Gaziantep University, Gaziantep 27310, Turkey; mmerkepci@gantep.edu.tr; \\ Tel.: +90-507-355-7515
}

Received: 22 July 2018; Accepted: 24 August 2018; Published: 27 August 2018

\begin{abstract}
Mathematical physics looks for ways to apply mathematical ideas to problems in physics. In differential forms, the tensor form is first defined, and the definitions of exterior and symmetric differential forms are made accordingly. For instance, $\mathrm{M}$ is an R-module, $M \otimes_{R} M$ the tensor product of $\mathrm{M}$ with itself and $\mathrm{H}$ a submodule of $M \otimes_{R} M$ generated by $x \otimes y-y \otimes x$, where $x, y$ in $\mathrm{M}$. Then, $\vee^{2}(M)=M \otimes_{R} M / H$ is called the second symmetric power of $\mathrm{M}$. A role of the exterior differential forms in field theory is related to the conservation laws for physical fields, etc. In this study, I present a new approach to emphasize the properties of second exterior and symmetric derivations on Kahler modules, and I find a connection between them. I constitute exact sequences of $\vee^{2}\left(\Omega_{1}(S)\right)$ and $\Lambda^{2}\left(\Omega_{1}(S)\right)$, and I describe and prove a new isomorphism in the following: Let $S$ be an affine algebra presented by $R / I$, where $R=k\left[x_{1}, \ldots x_{s}\right]$ is a polynomial algebra and $I=\left(f_{1}, \ldots f_{m}\right)$ an ideal of $R$. Then, I have $J_{1} \Omega_{1}(S) \simeq \Omega_{1}(S) \oplus \vee^{2}\left(\Omega_{1}(S)\right) \oplus \Lambda^{2}\left(\Omega_{1}(S)\right.$.
\end{abstract}

Keywords: universal module; differential operators; Kähler module; symmetric derivation; exterior derivation

\section{Introduction}

Mathematical physics seeks any approaches to carry out mathematical ideas in problems in physics. These two disciplines are very close, especially with differential forms. Indeed, in the past, it was difficult to classify Newton and Gauss as purely physicists or mathematicians. Mathematical physics has been quite closely related to ideas in calculus, especially those of differential equations. An easy, acute definition of differential forms, due to H. Flanders [1], is that differential forms are those found under the integral signs, which give the current state of the differential forms. We draw up tensor field, related to components a basis formed of tensor products of basis tangent vectors $e_{i}$ and one-forms $w_{i}$, as displayed: $T=T_{\ldots m n}^{i k \ldots} e_{i} \otimes e_{k} \otimes \ldots w^{m} \otimes w^{n} \otimes \ldots$ The components could be functions of the position. One may work in the natural bases for these spaces written, for coordinates $x^{i}, e_{i}=\frac{\partial}{\partial x^{i}}, w^{i}=d x^{i}$. Differential forms are presented as antisymmetric covariant tensor fields, namely fields that only the $w^{i}$ perform and the components are the antisymmetric basis written as $w^{i} \wedge w^{j} \wedge$ $w^{k}=\sum_{\pi}(-1)^{\pi} \pi\left[w^{i} \otimes w^{j} \otimes w^{k}\right]$ composed of the antisymmetric tensor product of $w^{i} . \pi$ represents a permutation of the $w^{i}$. The symbol $\wedge$ is the called wedge product. The exterior derivative $d$ is a map from $p$-forms to $(p+1)$-forms. If $\gamma=f d x^{i} \wedge d x^{j} \wedge \ldots$, then $d$ is defined by $d \gamma=\left(\frac{\partial f}{\partial x^{k}}\right) d x^{k} \wedge d x^{i} \wedge d x^{j} \wedge \ldots$, and the exterior derivative satisfies the Leibniz rule: $d(\alpha \wedge \beta)=d \alpha \wedge \beta+(-1)^{p} \alpha \wedge d \beta .(p=\operatorname{rank\alpha })$ in [2]. I now work with the set of differential forms on a commutative algebra. I give lemmas about second order exterior derivations and give definitions and lemmas about symmetric derivations of Kähler differentials, and I improve new approaches regarding second order exterior and symmetric derivatives of Kähler differentials. 


\section{Mathematical Background}

One of the methods used to prove algebraic sets and their coordinate results is to study the universal modules of differential operators. Thus, problems related to algebraic sets are transferred to the module theory. For example, if $\mathrm{p}$ is a point in the algebraic set $\mathrm{V}$, then under suitable conditions, it can be shown that $\mathrm{p}$ is a simple point of $\mathrm{V}$ if and only if $\Omega_{1}(R)$ is a free module including a local ring $R$ corresponding to the point $\mathrm{p}$ of $\mathrm{V}$, and $\Omega_{1}(R)$ is the universal module of derivations of $R$. Universal modules of differential operators propose an alternative solution to the available criteria that helps to understand whether a coordinate ring corresponding to an irreducible finitely-generated algebra is regular or not. Universal modules were first defined by Y. Nakai in 1960 [3]. He gave the necessary and sufficient condition for an affine domain to be regular and gave an alternative idea of the Jacobian criterion for regularity. Universal modules of high-order differential operators were first described by H. Osborn in 1967 [4]. Y. Nakai [5] extended the fundamental theorems for higher derivations and some properties of the module of high order differentials. R.G. Heynemann and M.E. Sweedler also studied the same notations in [6]. They mentioned differential operators on a commutative algebra, which extends the notion of derivations. J. Johnson presented Kähler differentials and differential algebra in [7]. Then, Erdogan studied universal modules of higher differential operators in 1993 [8]. The exterior and symmetric derivations of universal modules were also studied by Osborn [4], Hart [9,10], Erdogan [8], Olgun [11], Merkepçi and Olgun [12], Merkepçi and et al. [13], M.E. Sweedler [14] and Karakuş and et al [15]. Erdogan mentioned the second order exterior derivations of universal modules. Olgun gave the definition of generalized symmetric derivations on Kähler modules and gave some homological properties. Merkepçi and Olgun defined some split exact sequences and isomorphisms about second order symmetric and exterior derivations on Kähler modules, and they calculated the projective dimension of second order symmetric derivations on Kähler modules.

\section{Preliminaries}

Let $\mathrm{k}$ be a field with characteristic zero and $R$ be a commutative algebra over k. $J_{1}(R)$ is the universal module of first order differentials of $R$ over k. $\Omega_{1}(R)$ is the module of first order Kähler differentials of $R$ over k. $d_{1}$ is the first order k-derivation $R \longrightarrow \Omega_{1}(R)$ of $R$.

$\Lambda^{2}\left(\Omega_{1}(R)\right)$ is the second order exterior derivation of Kähler modules on $\Omega_{1}(R)$, and $\bigvee^{2}\left(\Omega_{1}(R)\right)$ is the second order symmetric derivation of Kähler modules on $\Omega_{1}(R)$.

Definition 1. Let $R$ be any $k$-algebra (commutative with unit), $R \rightarrow \Omega_{n}(R)$ be the $n$-th order Kähler derivation of $R$ and $\vee\left(\Omega_{n}(R)\right)$ be the symmetric algebra $\bigoplus_{p \geq 0} \vee^{p}\left(\Omega_{n}(R)\right)$ generated over $R$ by $\Omega_{n}(R)$ [2].

A generalized symmetric derivation is any $k$-linear map $D$ of $\vee\left(\Omega_{n}(R)\right)$ into itself such that:

(i) $D\left(\vee^{p}\left(\Omega_{n}(R)\right)\right) \subset \vee^{p+1}\left(\Omega_{n}(R)\right)$

(ii) $D$ is an $n$-th order derivation over $k$ and

(iii) the restriction of $D$ to $R\left(R \simeq \vee^{0}\left(\Omega_{n}(R)\right)\right)$ is the Kähler derivation $d_{n}: R \rightarrow \Omega_{n}(R)$.

Lemma 1. Let $R$ be a commutative k-algebra. Suppose that $\Omega_{1}(R)$ is the universal module of derivations of $R$ with universal derivation $d: R \rightarrow \Omega_{1}(R)$ [6]. Then, the map:

$$
\begin{gathered}
D: \Omega_{1}(R) \rightarrow \Lambda^{2}\left(\Omega_{1}(R)\right) \\
D\left(\Sigma_{i} a_{i} d\left(b_{i}\right)\right)=\Sigma_{i} d\left(a_{i}\right) \wedge d\left(b_{i}\right)
\end{gathered}
$$

is a differential operator of order 1 on $\Omega_{1}(R)$ where $a_{i}, b_{i}$ in $R$.

Proof of Lemma 1. (Osborn, H. Lemma (9.2). p. 155)

Proposition 1. Let $S$ be an affine algebra presented by $R / I$. Then, the map $g: \Lambda^{2}(F / N) \longrightarrow \Lambda^{2} F / L_{N}$ defined by $g\left(\overline{d_{1}\left(x_{i}\right)} \wedge \overline{d_{1}\left(x_{j}\right)}\right)=\overline{d_{1}\left(x_{i}\right) \wedge d_{1}\left(x_{j}\right)}$ is an isomorphism of $S$-modules, where $\Lambda^{2} F$ is a free S-module 
with basis $\left\{d_{1}\left(x_{i}\right) \wedge d_{1}\left(x_{j}\right): 1 \leq i<j \leq s\right\}$. $L_{N}$ is a submodule of $\Lambda^{2} F$ generated by the set $\left\{d\left(f_{k}\right) \wedge d\left(x_{j}\right)\right.$ : $k=1, \ldots, m ; j=1, \ldots, s\}$.

Proof of Proposition 1. This follows by Proposition 4.1.5 in [6].

\section{Main Results}

Lemma 2. Let $S$ be an affine algebra presented by $R / I$. Then,

$$
\Omega_{1}(S) \stackrel{\hat{\Delta}_{1}}{\longrightarrow} J_{1}\left(\Omega_{1}(S)\right) \stackrel{\hat{\beta}_{1}}{\longrightarrow} \Lambda^{2}\left(\Omega_{1}(S)\right) \longrightarrow 0
$$

is an exact sequence of S-modules.

Proof of Lemma 2. The following map:

$$
\begin{gathered}
\hat{D}_{1}: \Omega_{1}(S) \rightarrow \Lambda^{2}\left(\Omega_{1}(S)\right) \\
\hat{D}_{1}\left(\sum_{i, j} x_{i} d_{1}\left(x_{j}\right)\right)=\sum_{i, j} d_{1}\left(x_{i}\right) \wedge d_{1}\left(x_{j}\right)
\end{gathered}
$$

is a differential operator of order one on $\Omega_{1}(S)$ where $x_{i}, x_{j} \in R$ and $1 \leq i, j \leq s$.

By the universal property of $J_{1}\left(\Omega_{1}(S)\right)$, there is a unique R-module homomorphism:

$$
\hat{\beta}_{1}: J_{1}\left(\Omega_{1}(S)\right) \rightarrow \Lambda^{2}\left(\Omega_{1}(S)\right)
$$

such that $\hat{\beta}_{1} \hat{\Delta}_{1}=\hat{D}_{1}$ and the following diagram commutes.

$$
\begin{array}{ccc}
\Omega_{1}(S) & \stackrel{\hat{D}_{1}}{\longrightarrow} & \Lambda^{2}\left(\left(\Omega_{1}(S)\right)\right) \\
\hat{\Delta}_{1} \searrow & & \nearrow \exists ! \hat{\beta}_{1} \\
& J_{1}\left(\Omega_{1}(S)\right) &
\end{array}
$$

Since,

$$
\begin{aligned}
\hat{\beta}_{1} \hat{\Delta}_{1}\left(x_{i} d_{1}\left(x_{j}\right)\right) & =\hat{D}_{1}\left(x_{i} d_{1}\left(x_{j}\right)\right) \\
=d_{1}\left(x_{i}\right) & \wedge d_{1}\left(x_{j}\right)
\end{aligned}
$$

$\hat{\beta}_{1}$ is surjective. Therefore, we have:

$$
\Omega_{1}(S) \stackrel{\hat{\Delta}_{1}}{\longrightarrow} J_{1}\left(\Omega_{1}(S)\right) \stackrel{\hat{\beta}_{1}}{\longrightarrow} \Lambda^{2}\left(\Omega_{1}(S)\right) \longrightarrow 0
$$

an exact sequence of S-modules. It is sufficient to show that the sequence is exact at $J_{1}\left(\Omega_{1}(S)\right)$.

Note that $\operatorname{Im} \hat{\Delta}_{1}$ is generated by $\hat{\Delta}_{1}\left(d_{1}\left(x_{i}\right)\right)$ for $i=1, \ldots, \mathrm{s}$.

Therefore, we have:

$$
\hat{\beta}_{1} \hat{\Delta}_{1}\left(d_{1}\left(x_{i}\right)\right)=\hat{D}_{1}\left(d_{1}\left(x_{i}\right)\right)=d_{1}(1) \wedge d_{1}\left(x_{i}\right)=0
$$

Hence, $\operatorname{Im} \hat{\Delta}_{1}$ is in $\operatorname{ker} \hat{\beta}_{1}$. Therefore, we get $\hat{p}: J_{1}\left(\Omega_{1}(S)\right) / \operatorname{Im} \hat{\Delta}_{1} \rightarrow \Lambda^{2}\left(\Omega_{1}(S)\right)$ defined by:

$$
\hat{p} \overline{\left(\hat{\Delta}_{1}\left(x_{i} d_{1}\left(x_{j}\right)\right)\right.}=d_{1}\left(x_{i}\right) \wedge d_{1}\left(x_{j}\right)
$$

Now, assume that $\lambda^{2} F$ and $L_{N}$ are as in Proposition $1 .\left\{\hat{\Delta}_{1}\left(x_{i} d_{1}\left(x_{j}\right)\right): 1 \leq i<j \leq s\right\}$ generates $J_{1}\left(\Omega_{1}(S)\right) / \operatorname{Im} \hat{\Delta}_{1}$. Since $\Lambda^{2} F$ is a free S-module with basis $d_{1}\left(x_{i}\right) \wedge d_{1}\left(x_{j}\right)$, we can write a map $\hat{q}$ : $\Lambda^{2} F \longrightarrow J_{1}\left(\Omega_{1}(S)\right) / \operatorname{Im} \hat{\Delta_{1}}$ by: 


$$
\hat{q}\left(d_{1}\left(x_{i}\right) \wedge d_{1}\left(x_{j}\right)\right)=\overline{\hat{\Delta}_{1}\left(x_{i} d_{1}\left(x_{j}\right)\right)}
$$

Thus, if $\left\{f_{k}\right\}$ is a generating set for $\mathrm{I}$, we have:

$$
\hat{q}\left(d_{1}\left(f_{k}\right) \wedge d_{1}\left(x_{i}\right)\right)=\hat{q}\left(\sum_{i} \frac{\partial f_{k}}{\partial x_{i}} d_{1}\left(x_{i}\right) \wedge d_{1}\left(x_{i}\right)\right)=\sum_{i} \frac{\partial f_{k}}{\partial x_{i}} \hat{\Delta_{1}}\left(x_{i} d_{1}\left(x_{i}\right)=\hat{\Delta_{1}}\left(f_{k} d_{1}\left(x_{i}\right)\right)=o\right.
$$

where $\frac{\partial}{\partial x_{i}}: R \longrightarrow R, \partial_{i}\left(x_{j}\right)=\delta_{i}, j$ for $i, j=1, \ldots$, s and $\delta_{i}, j$ is the Kronecker delta. Hence, $\hat{q}\left(L_{N}\right)=0$.

Therefore, $\hat{q}$ induces an S-module homomorphism: $\hat{\hat{q}}: \Lambda^{2} F / L_{N} \longrightarrow J_{1}\left(\Omega_{1}(S)\right) / \operatorname{Im} \hat{\Delta_{1}}$

$$
\overline{\hat{q}}\left(\overline{d_{1}\left(x_{i}\right) \wedge d_{1}\left(x_{j}\right)}\right)=\overline{\Delta_{1}\left(x_{i} d_{1}\left(x_{j}\right)\right)}
$$

It is clear that $\bar{q} \hat{p}$ and $\hat{p} \hat{q}$ are identities, and so, $\operatorname{ker} \hat{p}=\operatorname{ker} \hat{\beta}_{1} / \operatorname{Im} \hat{\Delta}_{1}=0$ and then $\operatorname{ker} \hat{\beta}_{1}=\operatorname{Im} \hat{\Delta}_{1}$.

Therefore, the sequence is exact. Similarly, the following lemma is given.

Lemma 3. Let $S$ be an affine algebra presented by R/I. Then:

$$
\Omega_{1}(S) \stackrel{\check{\Delta}_{1}}{\longrightarrow} J_{1}\left(\Omega_{1}(S)\right) \stackrel{\check{\beta}_{1}}{\longrightarrow} \bigvee^{2}\left(\Omega_{1}(S)\right) \longrightarrow 0
$$

is an exact sequence of S-modules.

Proof of Lemma 3. The following map:

$$
\begin{aligned}
& \check{D_{1}}: \Omega_{1}(S) \rightarrow \vee^{2}\left(\Omega_{1}(S)\right) \\
& \check{D_{1}}\left(\sum_{i, j} x_{i} d_{1}\left(x_{j}\right)\right)=\sum_{i, j} d_{1}\left(x_{i}\right) \vee d_{1}\left(x_{j}\right)
\end{aligned}
$$

is a differential operator of order one on $\Omega_{1}(S)$ where $x_{i}, x_{j}$ in $R$ and $1 \leq i, j \leq s$. By the universal mapping property of $J_{1}\left(\Omega_{1}(S)\right)$, there is a unique S-module homomorphism $\check{\beta}_{1}: J_{1}\left(\Omega_{1}(S)\right) \longrightarrow$ $\vee^{2}\left(\Omega_{1}(S)\right)$ such that $\breve{\beta}_{1} \breve{\Delta}_{1}=\breve{D}_{1}$ and the following diagram commutes.

$$
\begin{array}{ccc}
\Omega_{1}(S) & \stackrel{\check{D}_{1}}{\longrightarrow} & V^{2}\left(\Omega_{1}(S)\right) \\
\check{\Delta}_{1} \searrow & & \nearrow \exists ! \check{\beta}_{1} \\
& J_{1}\left(\Omega_{1}(S)\right) &
\end{array}
$$

Since,

$$
\check{\beta}_{1}\left(\check{\Delta}_{1}\left(x_{i} d_{1}\left(x_{j}\right)\right)\right)=\check{D}_{1}\left(x_{i} d_{1}\left(x_{j}\right)\right)=d_{1}\left(x_{i}\right) \vee d_{1}\left(x_{j}\right)
$$

$\check{\beta}_{1}$ is surjective. Therefore, we have:

$$
\Omega_{1}(S) \stackrel{\check{\Delta}_{1}}{\longrightarrow} J_{1}\left(\Omega_{1}(S)\right) \stackrel{\check{\beta}_{1}}{\longrightarrow} \bigvee^{2}\left(\Omega_{1}(S)\right) \longrightarrow 0
$$

an exact sequence of S-modules. It is sufficient to prove that the sequence is exact at $J_{1}\left(\Omega_{1}(S)\right)$.

Note that, $\operatorname{Im} \breve{\Delta}_{1}$ is generated by $\check{\Delta}_{1}\left(d_{1}\left(x_{i}\right)\right)$, for $i=1, \ldots$, s. Therefore, we have:

$$
\check{\beta}_{1} \check{\Delta}_{1}\left(d_{1}\left(x_{i}\right)\right)=\check{D}_{1}\left(d_{1}\left(x_{i}\right)\right)=d_{1}(1) \vee d_{1}\left(x_{i}\right)=0
$$

It shows that $\operatorname{Im} \check{\Delta}_{1}$ is in $\operatorname{ker} \check{\beta}_{1}$. Therefore, we get $\check{p}: J_{1}\left(\Omega_{1}(S)\right) / \operatorname{Im} \check{\Delta}_{1} \rightarrow V^{2}\left(\Omega_{1}(S)\right)$ defined by:

$$
\check{p} \overline{\left(\check{\Delta}_{1}\left(x_{i} d_{1}\left(x_{j}\right)\right)\right.}=d_{1}\left(x_{i}\right) \vee d_{1}\left(x_{j}\right)
$$


Now, assume that $\bigvee^{2} F$ and $L_{N}$ are as in Proposition 1. $\left\{\check{\Delta}_{1}\left(x_{i} d_{1}\left(x_{j}\right)\right) \mid 1 \leq i<j \leq s\right\}$ generates $J_{1}\left(\Omega_{1}(S)\right) / \operatorname{Im} \breve{\Delta}_{1}$. Since $\vee^{2} F$ is a free S-module with basis $d_{1}\left(x_{i}\right) \vee d_{1}\left(x_{j}\right)$, we can write a map $\check{q}$ : $\vee^{2} F \longrightarrow J_{1}\left(\Omega_{1}(S)\right) / \operatorname{Im} \check{\Delta_{1}}$ by:

$$
\breve{q}\left(d_{1}\left(x_{i}\right) \vee d_{1}\left(x_{j}\right)\right)=\overline{\check{\Delta}_{1}\left(x_{i} d_{1}\left(x_{j}\right)\right)}
$$

Thus, if $\left\{f_{k}\right\}$ is a generating set for $\mathrm{I}$, we have:

$$
\check{q}\left(d_{1}\left(f_{k}\right) \vee d_{1}\left(x_{i}\right)\right)=\check{q}\left(\sum_{i} \frac{\partial f_{k}}{\partial x_{i}} d_{1}\left(x_{i}\right) \vee d_{1}\left(x_{i}\right)\right)=\sum_{i} \frac{\partial f_{k}}{\partial x_{i}} \check{\Delta}_{1}\left(x_{i} d_{1}\left(x_{i}\right)=\check{\Delta_{1}}\left(f_{k} d_{1}\left(x_{i}\right)\right)=o\right.
$$

where $\frac{\partial}{\partial x_{i}}: R \longrightarrow R, \partial_{i}\left(x_{j}\right)=\delta_{i}, j$ for $i, j=1, \ldots$, s and $\delta_{i}, j$ is the Kronecker delta. Hence, $\breve{q}\left(L_{N}\right)=0$.

Therefore, $\check{q}$ induces an S-module homomorphism $\bar{q}: \vee^{2} F / L_{N} \longrightarrow J_{1}\left(\Omega_{1}(S)\right) / \operatorname{Im} \check{\Delta_{1}}$ :

$$
\bar{q}\left(\overline{d_{1}\left(x_{i}\right) \vee d_{1}\left(x_{j}\right)}\right)=\overline{\Delta_{1}\left(x_{i} d_{1}\left(x_{j}\right)\right)}
$$

It is clear that $\bar{q} \breve{p}$ and $\breve{p} \bar{q}$ are identities, and so, $\operatorname{ker} \check{p}=\operatorname{ker} \check{\beta}_{1} / \operatorname{Im} \check{\Delta}_{1}=0$ and then $\operatorname{ker} \check{\beta}_{1}=\operatorname{Im} \check{\Delta}_{1}$.

Therefore, the sequence is exact.

Theorem 1. $S$ is an affine algebra presented by R/I. Then, there exists a split short exact sequence of S-modules:

$$
0 \longrightarrow \operatorname{ker} \beta \longrightarrow J_{1}\left(\Omega_{1}(S)\right) \stackrel{\beta}{\longrightarrow} \Omega_{1}(S) \longrightarrow 0
$$

and

$$
k e r \beta \simeq \Lambda^{2}\left(\Omega_{1}(S)\right) \oplus \vee^{2}\left(\Omega_{1}(S)\right)
$$

so, we have:

$$
J_{1}\left(\Omega_{1}(S)\right) \simeq \Omega_{1}(S) \oplus \Lambda^{2}\left(\Omega_{1}(S)\right) \oplus \vee^{2}\left(\Omega_{1}(S)\right)
$$

Proof of Theorem 1. The following diagram commutes such that $\beta \Delta_{1}=1_{S}$.

$$
\begin{array}{lll}
\Omega_{1}(S) & \stackrel{1_{S}}{\longrightarrow} & \Omega_{1}(S) \\
\Delta_{1} \searrow & & \nearrow \exists ! \beta
\end{array}
$$

Since,

$$
\beta\left(\Delta_{1}\left(\sum_{i, j} x_{i} d_{1}\left(x_{j}\right)\right)\right)=\sum_{i, j}\left(x_{i}\right) d_{1}\left(x_{j}\right)
$$

in $\Omega_{1}(S)$ for $i, j=1, \ldots, \mathrm{s}$.

$\beta$ is surjective. Therefore, we have:

$$
0 \longrightarrow \operatorname{ker} \beta \longrightarrow J_{1}\left(\Omega_{1}(S)\right) \stackrel{\beta}{\longrightarrow} \Omega_{1}(S) \rightarrow 0
$$

a short exact sequence of S-modules.

Now, the map:

$$
\hat{i}: \Lambda^{2}\left(\Omega_{1}(S)\right) \longrightarrow J_{1}\left(\Omega_{1}(S)\right)
$$

defined by:

$$
\begin{gathered}
\hat{i}\left(d_{1}\left(x_{i}\right) \wedge d_{1}\left(x_{j}\right)\right)=1 / 2\left[\Delta_{1}\left(x_{i} d_{1}\left(x_{j}\right)\right)+\Delta_{1}\left(x_{j} d_{1}\left(x_{i}\right)\right.\right. \\
\left.+x_{i} \Delta_{1}\left(d_{1}\left(x_{j}\right)\right)-x_{j} \Delta_{1} d_{1}\left(x_{i}\right)\right]
\end{gathered}
$$

Similarly, the map: 


$$
\check{i}: \bigvee^{2}\left(\Omega_{1}(S)\right) \longrightarrow J_{1}\left(\Omega_{1}(S)\right)
$$

defined by:

$$
\begin{gathered}
\check{i}\left(d_{1}\left(x_{i}\right) \vee d_{1}\left(x_{j}\right)\right)=1 / 2\left[\Delta_{1}\left(x_{i} d_{1}\left(x_{j}\right)\right)-\Delta_{1}\left(x_{j} d_{1}\left(x_{i}\right)\right)\right. \\
\left.-x_{i} \Delta_{1}\left(d_{1}\left(x_{j}\right)\right)+x_{j} \Delta_{1} d_{1}\left(x_{i}\right)\right]
\end{gathered}
$$

Then, we define the following map:

$$
\begin{gathered}
g: \Lambda^{2}\left(\Omega_{1}(S)\right) \oplus \vee^{2}\left(\Omega_{1}(S)\right) \longrightarrow J_{1}\left(\Omega_{1}(S)\right) \\
g(x, y)=\beta \hat{i}(x)+\beta \check{i}(y)
\end{gathered}
$$

$x \in \Lambda^{2}\left(\Omega_{1}(S)\right)$ and $y \in \bigvee^{2}\left(\Omega_{1}(S)\right)$.

Therefore,

$$
k e r \beta \cong \Lambda^{2}\left(\Omega_{1}(S)\right) \oplus \vee^{2}\left(\Omega_{1}(S)\right)
$$

Shortly, I prove the splitting. Let:

$$
\hat{D_{1}}: \Omega_{1}(S) \longrightarrow \Lambda^{2}\left(\Omega_{1}(S)\right)
$$

by:

$$
\hat{D_{1}}\left(\sum_{i, j} x_{i} d_{1}\left(x_{j}\right)\right)=d_{1}\left(x_{i}\right) \wedge d_{1}\left(x_{j}\right)
$$

and:

$$
\check{D_{1}}: \Omega_{1}(S) \longrightarrow \vee^{2}\left(\Omega_{1}(S)\right)
$$

defined by:

$$
\breve{D_{1}}\left(\sum_{i, j} x_{i} d_{1}\left(x_{j}\right)\right)=d_{1}\left(x_{i}\right) \vee d_{1}\left(x_{j}\right)
$$

$\hat{D}_{1}$ and $\check{D}_{1}$ are first order derivations.

Therefore, by the universal property of $J_{1}\left(\Omega_{1}(S)\right)$, there exists S-module homomorphisms:

$$
\begin{aligned}
& \hat{\beta}_{1}: J_{1}\left(\Omega_{1}(S)\right) \longrightarrow \Lambda^{2}\left(\Omega_{1}(S)\right) \\
& \check{\beta}_{1}: J_{1}\left(\Omega_{1}(S)\right) \longrightarrow \vee^{2}\left(\Omega_{1}(S)\right)
\end{aligned}
$$

such that diagrams:

$$
\begin{array}{cccc}
\Omega_{1}(S) & & \stackrel{\hat{D}_{1}}{\longrightarrow} & \Lambda^{2}\left(\Omega_{1}(S)\right) \\
\downarrow \Delta_{1} & & & \downarrow 1_{\Omega_{1}(S)} \\
J_{1}\left(\Omega_{1}(S)\right) & \stackrel{\hat{\beta}_{1}}{\longrightarrow} & \Lambda^{2}\left(\Omega_{1}(S)\right)
\end{array}
$$

and:

$$
\begin{array}{cccc}
\Omega_{1}(S) & \stackrel{\check{D_{1}}}{\longrightarrow} & \vee^{2}\left(\Omega_{1}(S)\right) \\
\downarrow \Delta_{1} & & & \downarrow 1_{\Omega_{1}(S)} \\
J_{1}\left(\Omega_{1}(S)\right) & \stackrel{\check{\beta}_{1}}{\longrightarrow} & \vee^{2}\left(\Omega_{1}(S)\right)
\end{array}
$$

commutes.

Therefore, I can write: 


$$
\hat{\beta}_{1} \Delta_{1}\left(\sum_{i, j} x_{i} d_{1}\left(x_{j}\right)\right)=\hat{D}_{1}\left(\sum_{i, j} x_{i} d_{1}\left(x_{j}\right)\right)=\sum_{i, j} d_{1}\left(x_{i}\right) \wedge d_{1}\left(x_{j}\right)
$$

and:

$$
\check{\beta}_{1} \Delta_{1}\left(\sum_{i, j} x_{i} d_{1}\left(x_{j}\right)\right)=\check{D}_{1}\left(\sum_{i, j} x_{i} d_{1}\left(x_{j}\right)\right)=\sum_{i, j} d_{1}\left(x_{i}\right) \vee d_{1}\left(x_{j}\right)
$$

From here, I obtain:

$$
\begin{gathered}
\hat{\beta}_{1} \hat{i}=1_{\Lambda^{2}\left(\Omega_{1}(S)\right)} \\
\check{\beta}_{1} \check{i}=1_{\bigvee^{2}\left(\Omega_{1}(S)\right)} \\
\hat{\beta}_{1} \check{i}=0 \\
\check{\beta}_{1} \hat{i}=0
\end{gathered}
$$

Hence, the sequence splits.

Example 1. Let $R=k\left[x_{1}, x_{2}, x_{3}\right]$ be a polynomial algebra of dimension three. Then, $\Omega_{1}(R)$ is a free $R$-module of rank $\left(\begin{array}{l}4 \\ 1\end{array}\right)-1=3$ with basis $\left\{d_{1}\left(x_{1}\right), d_{1}\left(x_{2}\right), d_{1}\left(x_{3}\right)\right\}$, and $\vee^{2}\left(\Omega_{1}(R)\right)$ is a free $R$-module of rank $\left(\begin{array}{l}4 \\ 2\end{array}\right)=6$ with basis $\left\{d_{1}\left(x_{1}\right) \vee d_{1}\left(x_{1}\right), d_{1}\left(x_{1}\right) \vee d_{1}\left(x_{2}\right), d_{1}\left(x_{1}\right) \vee d_{1}\left(x_{3}\right), d_{1}\left(x_{2}\right) \vee d_{1}\left(x_{2}\right), d_{1}\left(x_{2}\right) \vee d_{1}\left(x_{3}\right), d_{1}\left(x_{3}\right) \vee\right.$ $\left.d_{1}\left(x_{3}\right)\right\} . \quad \Lambda^{2}\left(\Omega_{1}(R)\right)$ is a free $R$-module of rank $\left(\begin{array}{l}3 \\ 2\end{array}\right)=3$ with basis $\left\{d_{1}\left(x_{1}\right) \wedge d_{1}\left(x_{2}\right), d_{1}\left(x_{1}\right) \wedge\right.$ $\left.d_{1}\left(x_{3}\right), d_{1}\left(x_{2}\right) \wedge d_{1}\left(x_{3}\right)\right\}$, and $J_{1}\left(\Omega_{1}(R)\right)$ is generated by:

$\left\{\Delta_{1}\left(d_{1}\left(x_{1}\right)\right), \Delta_{1}\left(d_{1}\left(x_{2}\right)\right), \Delta_{1}\left(d_{1}\left(x_{3}\right)\right), \Delta_{1}\left(x_{1} d_{1}\left(x_{1}\right)\right), \Delta_{1}\left(x_{1} d_{1}\left(x_{2}\right)\right), \Delta_{1}\left(x_{1} d_{1}\left(x_{3}\right)\right), \Delta_{1}\left(x_{2} d_{1}\left(x_{1}\right)\right), \Delta_{1}\left(x_{2} d_{1}\left(x_{2}\right)\right)\right.$,

$$
\left.\Delta_{1}\left(x_{2} d_{1}\left(x_{3}\right)\right), \Delta_{1}\left(x_{3} d_{1}\left(x_{1}\right)\right), \Delta_{1}\left(x_{3} d_{1}\left(x_{2}\right)\right), \Delta_{1}\left(x_{3} d_{1}\left(x_{3}\right)\right)\right\}
$$

so, I identify isomorphism from ranks of $\left.\Omega_{1}(R), V^{2} \Omega_{1}(R)\right), \Lambda^{2} \Omega_{1}(R)$. Therefore,

$$
J_{1}\left(\Omega_{1}(R)\right) \cong \Omega_{1}(R) \oplus \vee^{2}\left(\Omega_{1}(R)\right) \oplus \Lambda^{2}\left(\Omega_{1}(R)\right)
$$

Example 2. Let $S$ be the coordinate ring of the cups $x_{2} x_{3}=x_{1}^{3}$. Then, $S=k\left[x_{1}, x_{2}, x_{3}\right] /(f)$ where $f=x_{2} x_{3}-x_{1}^{3} . \Omega_{1}(S) \cong F / N$ whereF is a free $S$-module on $\left\{d_{1}\left(x_{1}\right), d_{1}\left(x_{2}\right), d_{1}\left(x_{3}\right)\right\}$ and $N$ is a submodule of $F$ generated by $d_{1}(f)=x_{2} d_{1}\left(x_{3}\right)+x_{3} d_{1}\left(x_{2}\right)-3 x_{1}^{2} d_{1}\left(x_{1}\right)$. Since rank $\left(\Omega_{1}(S)\right)=\left(\begin{array}{c}1+2 \\ 2\end{array}\right)-1=2$,

$I$ have rank $N=\operatorname{rankF}-\operatorname{rank} \Omega_{1}(S)=3-2=1$. Therefore, $N$ is free S-module. Similarly, $\vee^{2}\left(\Omega_{1}(S)\right) \cong \vee^{2} F / L_{N}$ where $\vee^{2} F$ is a free module with basis $\left\{d_{1}\left(x_{1}\right) \vee d_{1}\left(x_{1}\right), d_{1}\left(x_{1}\right) \vee\right.$ $\left.d_{1}\left(x_{2}\right), d_{1}\left(x_{1}\right) \vee d_{1}\left(x_{3}\right), d_{1}\left(x_{2}\right) \vee d_{1}\left(x_{2}\right), d_{1}\left(x_{2}\right) \vee d_{1}\left(x_{3}\right), d_{1}\left(x_{3}\right) \vee d_{1}\left(x_{3}\right)\right\}$, and $L_{N}$ is a submodule of $\vee^{2} F$ generated by:

$$
\begin{aligned}
& d_{1}(f) \vee d_{1}\left(x_{1}\right)=x_{2} d_{1}\left(x_{3}\right) \vee d_{1}\left(x_{1}\right)+x_{3} d_{1}\left(x_{2}\right) \vee d_{1}\left(x_{1}\right)-3 x^{2} d_{1}\left(x_{1}\right) \vee d_{1}\left(x_{1}\right) \\
& d_{1}(f) \vee d_{1}\left(x_{2}\right)=x_{2} d_{1}\left(x_{3}\right) \vee d_{1}\left(x_{2}\right)+x_{3} d_{1}\left(x_{2}\right) \vee d_{1}\left(x_{2}\right)-3 x^{2} d_{1}\left(x_{1}\right) \vee d_{1}\left(x_{2}\right) \\
& d_{1}(f) \vee d_{1}\left(x_{3}\right)=x_{2} d_{1}\left(x_{3}\right) \vee d_{1}\left(x_{3}\right)+x_{3} d_{1}\left(x_{2}\right) \vee d_{1}\left(x_{3}\right)-3 x^{2} d_{1}\left(x_{1}\right) \vee d_{1}\left(x_{3}\right)
\end{aligned}
$$

$\operatorname{rank} \vee^{2}\left(\Omega_{1}(S)\right)=\left(\begin{array}{l}2+1 \\ 2-1\end{array}\right)=\left(\begin{array}{l}3 \\ 1\end{array}\right)=3$.

By the same argument, $\Lambda^{2}\left(\Omega_{1}(S)\right) \cong \Lambda^{2} F / l_{N}$ where $\Lambda^{2} F$ is a free module with basis $\left\{d_{1}\left(x_{1}\right) \wedge\right.$ $\left.d_{1}\left(x_{2}\right), d_{1}\left(x_{1}\right) \wedge d_{1}\left(x_{3}\right), d_{1}\left(x_{2}\right) \wedge d_{1}\left(x_{3}\right)\right\}$, and $l_{N}$ is a submodule of $\Lambda^{2} F$ generated by:

$$
\begin{aligned}
& d_{1}(f) \wedge d_{1}\left(x_{1}\right)=x_{2} d_{1}\left(x_{3}\right) \wedge d_{1}\left(x_{1}\right)+x_{3} d_{1}\left(x_{2}\right) \wedge d_{1}\left(x_{1}\right) \\
& d_{1}(f) \wedge d_{1}\left(x_{2}\right)=x_{2} d_{1}\left(x_{3}\right) \wedge d_{1}\left(x_{2}\right)-3 x_{1}^{2} d_{1}\left(x_{1}\right) \wedge d_{1}\left(x_{2}\right) \\
& d_{1}(f) \wedge d_{1}\left(x_{3}\right)=x_{3} d_{1}\left(x_{2}\right) \wedge d_{1}\left(x_{3}\right)-3 x_{1}^{2} d_{1}\left(x_{1}\right) \wedge d_{1}\left(x_{3}\right)
\end{aligned}
$$


$\operatorname{rank} \Lambda^{2}\left(\Omega_{1}(S)\right)=\left(\begin{array}{l}2 \\ 2\end{array}\right)=1$.

We derive the rank of $J_{1}\left(\Omega_{1}(S)\right)$ from the ranks of $\Omega_{1}(S), \vee^{2}\left(\Omega_{1}(S)\right), \Lambda^{2}\left(\Omega_{1}(S)\right)$, so $J_{1}\left(\Omega_{1}(S)\right) \cong \Omega_{1}(S) \oplus \Lambda^{2}\left(\Omega_{1}(S)\right) \oplus \vee^{2}\left(\Omega_{1}(S)\right)$.

\section{Discussion}

There are many studies about symmetric and exterior derivations of Kähler modules. Moreover, exterior derivations have been studied more extensively. Especially, there are articles about first order symmetric and exterior derivations of Kähler modules. Then, the question comes to mind: Is there a connection between these two subjects that are working in the same field? In this study, I examined the relationship between the symmetric and exterior derivations of Kähler modules. I am sure that the results that I have found are useful, especially in the next studies. Furthermore, I think the following questions are necessary for the future research:

(1) Can I define a new approach related to high order symmetric and exterior derivations of Kähler modules?

(2) Under which conditions can I write a connection about high order symmetric and exterior derivations on Kähler modules.

\section{Conclusions}

I know that symmetric and exterior derivations on Kähler modules play an important role both in mathematical physics and in commutative algebra. Studies on the structure of the symmetric and exterior derivations of Kähler modules present interesting aspects for not only mathematical physics, but also commutative algebra. Therefore, in this paper, I searched for new approaches for the connection of first order symmetric and exterior derivations. Finally, I defined some isomorphisms, proved them and gave special examples.

Funding: This research received no external funding.

Conflicts of Interest: The author declares no conflict of interest.

\section{References}

1. Flanders, H. Differential Forms; Academic Press: New York, NY, USA, 1963; Volume 1.

2. Harrison, B.K. Differential form symmetry analysis of two equations cited by Fushchych. In Proceedings of the Second International Conference on Symmetry in Nonlinear Mathematical Physics, Kyiv, Ukraine, 7-13 July 1997; Volume 1, pp. 21-33.

3. Nakai, Y. On the theory of differentials in commutative rings. J. Math. Soc. Jpn. 1961, 13, 63-84. [CrossRef]

4. Osborn, H. Modules of differentials II. Math. Ann. 1968, 175, 146-158. [CrossRef]

5. Nakai, Y. High order derivations. Osaka J. Math. 1970, 7, 1-27.

6. Sweedler, M.E.; Heyneman, R.G. Affine Hopf algebras. J. Algebra 1969, 13, 192-241.

7. Johnson J. Kähler Differentials and differential algebra. Ann. Math. 1969, 89, 92-98. [CrossRef]

8. Erdogan, A. Differential Operators and Their Universal Modules. Ph.D. Thesis, Universty of Leeds, Leeds, UK, 1993.

9. Hart, R. Higher derivations and universal differentials. J. Algebra 1996, 184, 175-181. [CrossRef]

10. Hart, R. Differential operators and affine algebra. J. Lond. Math. Soc. 1988, 28, 470-476. [CrossRef]

11. Olgun, N. A Problem on universal modules. Commun. Algebra 2015, 43, 4350-4358. [CrossRef]

12. Merkepci, H.; Olgun, N. Some results on Kähler modules. Algebra Lett. 2017, 2017, 5.

13. Merkepci, H.; Olgun, N.; Aydın, E. Projective dimension of second order symmetric derivations of Kähler modules for hypersurfaces. Algebra Lett. 2017, 2017, 6 . 
14. Sweedler, M.E. Right derivations and right differential operators. Pac. J. Math. 1980, 86, 327-360. [CrossRef]

15. Karakuş, A.; Olgun, N.; Şahin, M. On the second-degree exterior derivation of Kähler modules on $X \otimes Y$. Symmetry 2018, 10, 166. [CrossRef]

(C) 2018 by the authors. Licensee MDPI, Basel, Switzerland. This article is an open access article distributed under the terms and conditions of the Creative Commons Attribution (CC BY) license (http://creativecommons.org/licenses/by/4.0/). 